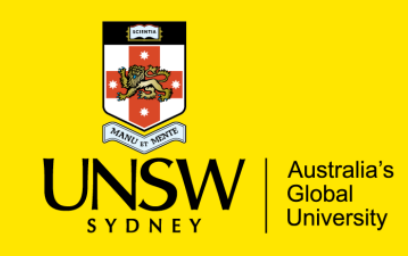

Presented by

Prof Andrew Dempster
Australian Centre for Space Engineering Research (ACSER)

\section{Use of GNSS Data as Evidence}




\section{Sources}

Andrew Dempster, "Use of GPS Data as Evidence in Court", Proc IGNSS 2018, Sydney 7-9 Feb 2018

Andrew G Dempster "GNSS Data as Court

Evidence: Lessons from Remote Sensing", Proc ION-GNSS+, Miami, 26-28 Sep 2018

Andrew Dempster, Allison Keally, Gary Edmond, "Questions for Providers of Expert Opinion on Logged GNSS Evidence", in preparation (abstract submitted to ION ITM, Reston, VA, Jan 2019) 


\section{Legal Concerns}

Early days: liability

- "Space Law treaties cannot solve liability questions about the failure of a GNSS signal"

- ICAO has tried to create a treaty re GNSS liability: not yet

- Galileo used SA as a lever

- (ubiquity $\rightarrow$ liable when GNSS not used..) 


\section{Legal Concerns}

Specifically to do with GPS "errors":

- Police forcing entry to the wrong home

- Repossession of wrong house

- Demolition of wrong house

Recent study (83 cases)

K J Berman, W B Glisson \& L M Glisson, "Investigating the Impact of Global Positioning System Evidence", 48th Hawaii International Conference on System Sciences, pp5234- 5243, 2015

- 19 criminal/ 11 civil classifications

- weight given to GNSS data "high" (8\%) or "medium" (54\%)

- Significant majority "admissible"

UNSW UG Solange Cunin Thesis - update for Aus

- What questions are asked in the cases? 


\section{Our Issue: Quality}

"the prosecution service needs to examine the GPS evidence thoroughly and must present other supporting evidence for GPS evidence to be admissible evidence in court"

Ishwar Khadka, "The accuracy of location services and the potential impact on the admissibility of GPS based evidence in court cases", BSc(Hons) thesis, University of

Derby, 2015 


\section{Aims}

Provide guidance for expert witnesses giving opinions on GNSS data

Recommend a standard for GNSS data logging 


\section{Motivating Example}

The Queen v Shane Anthony Day, 2014/00075246, NSW District Court

Mona Vale Road - dangerous driving causing death Was the driver speeding? 


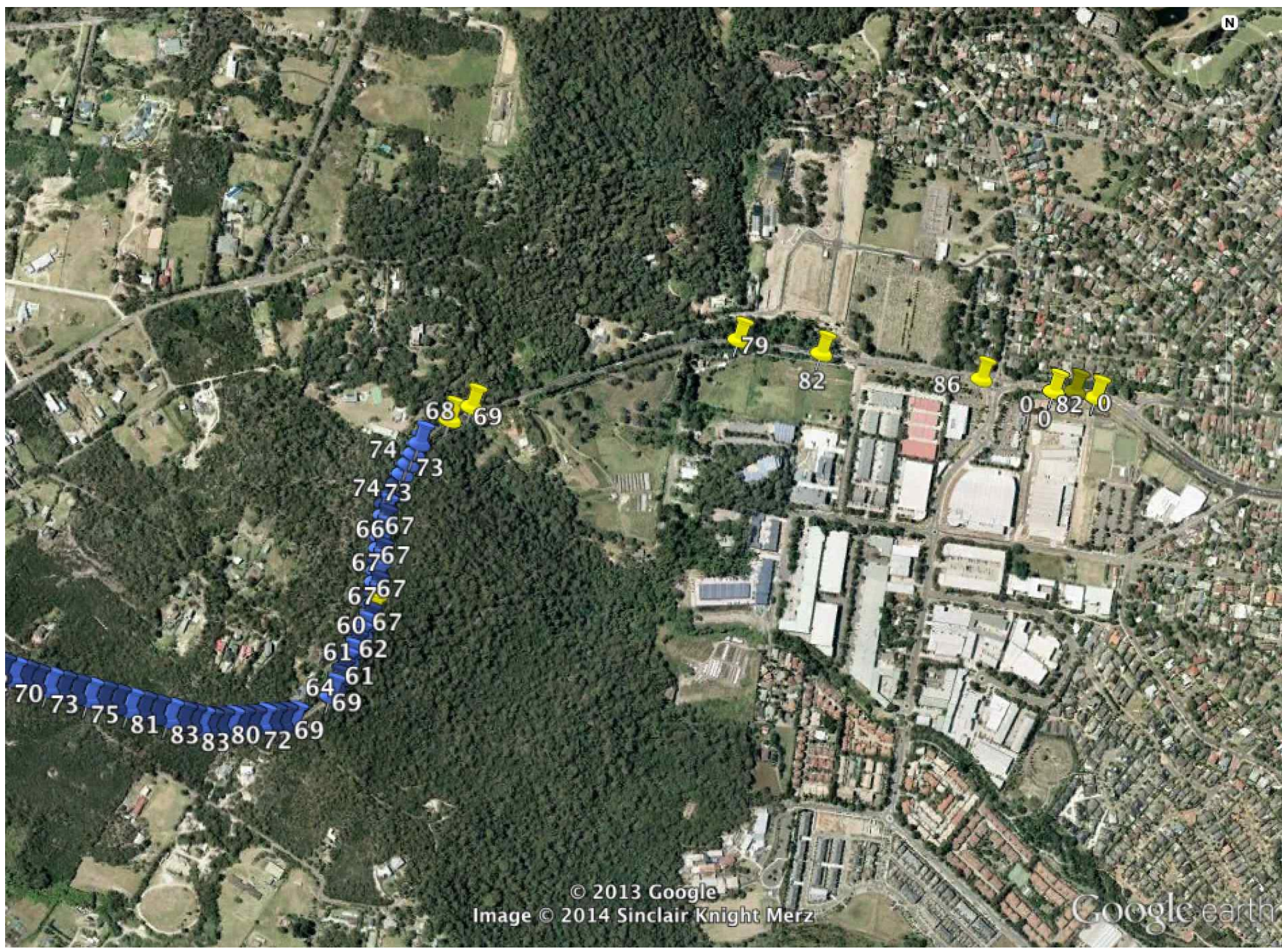

Slide 7

() ACSER

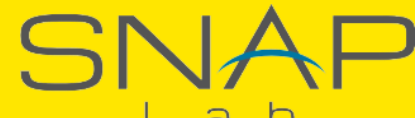

UNSW 


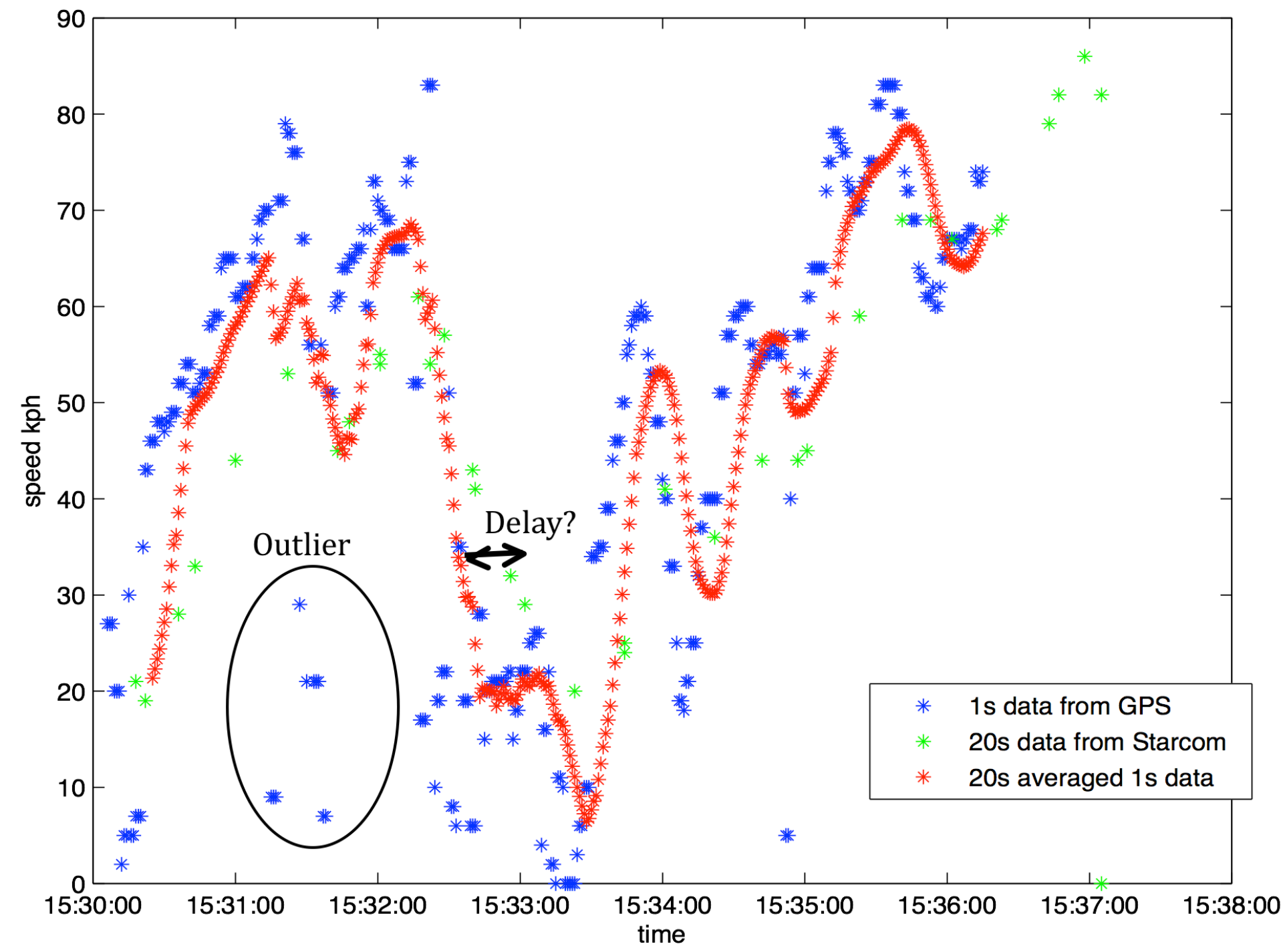




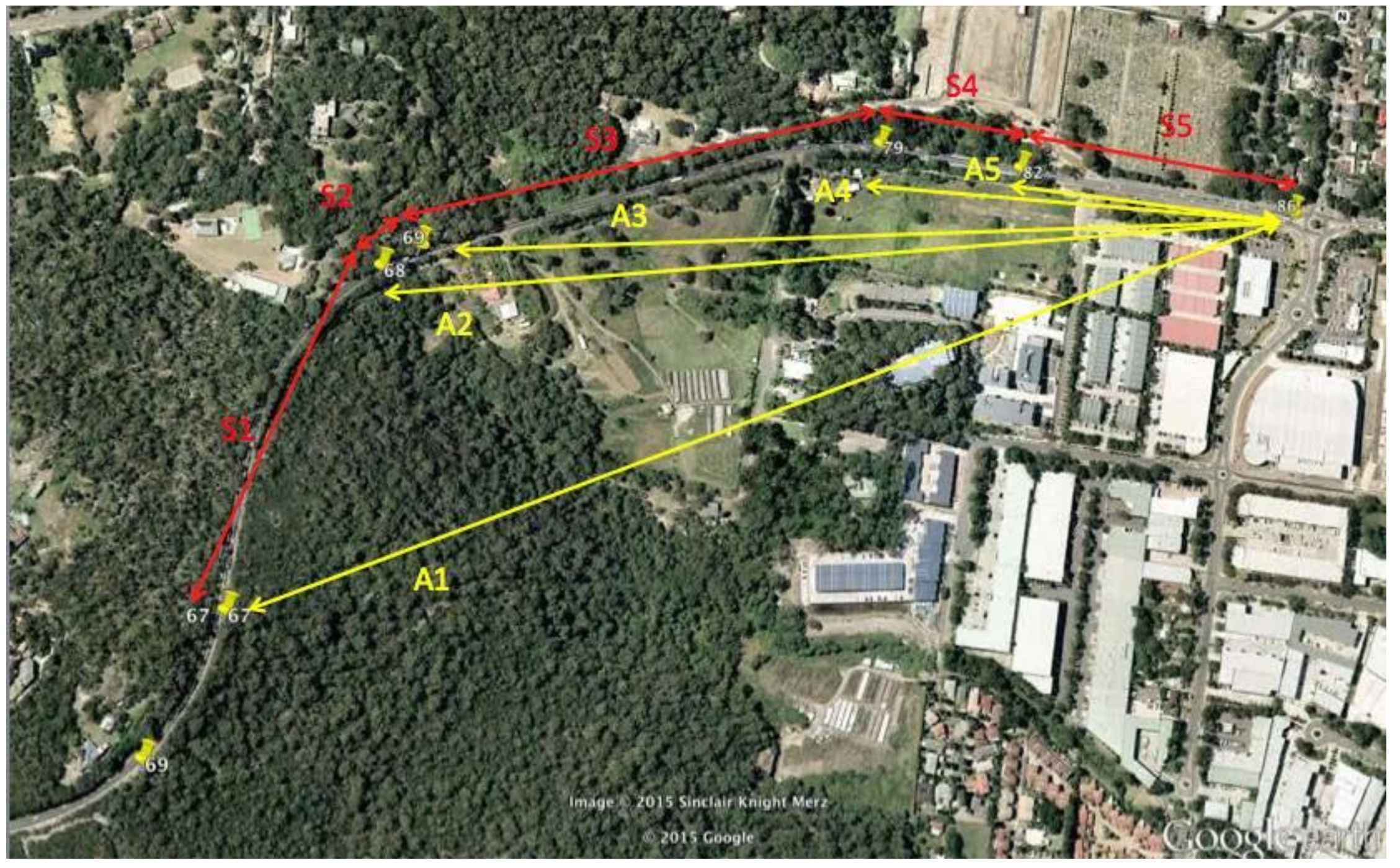

() ACSER

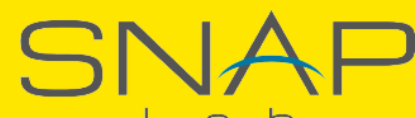

UNSW 


\begin{tabular}{|l|l|l|}
\hline Sector & Length $(\mathrm{m})$ & Speed $(\mathrm{kph})$ \\
\hline S1 & 358 & 72 \\
\hline S2 & 43 & 78 \\
\hline S3 & 482 & 87 \\
\hline S4 & 150 & 108 \\
\hline S5 & 292 & 96 \\
\hline A1 & 1148 & 74 \\
\hline A2 & 944 & 89 \\
\hline A3 & 905 & 90 \\
\hline A4 & 442 & 100 \\
\hline A5 & 292 & 96 \\
\hline
\end{tabular}

Slide 10 


\section{What to Learn From This Case Study lin questions:}

can you trust GNSS data?

if not, what would make you trust it? 


\section{Questions}

\section{Different questions for the receiver and the expert witness}

\begin{tabular}{|c|l|l|}
\hline Examples & Questions for GPS Receiver & Questions for Expert Witness \\
\hline Case Study 1 [2] & How fast was the vehicle going? & $\begin{array}{l}\text { How fast was the vehicle going? } \\
\text { Was it exceeding the speed limit? } \\
\text { How valid is the GPS data? }\end{array}$ \\
\hline Case Study 2 [2] & Where was the user? & How accurate was GPS at that time and place? \\
\hline Both examples in [7] & How fast was the vehicle going? & Did the vehicle exceed the speed limit? \\
\hline & & \\
\hline
\end{tabular}

Table 1 Different questions asked of GNSS receivers and relevant expert witness in case studies in [2] [7] 


\section{Sources of Problems: GNSS}

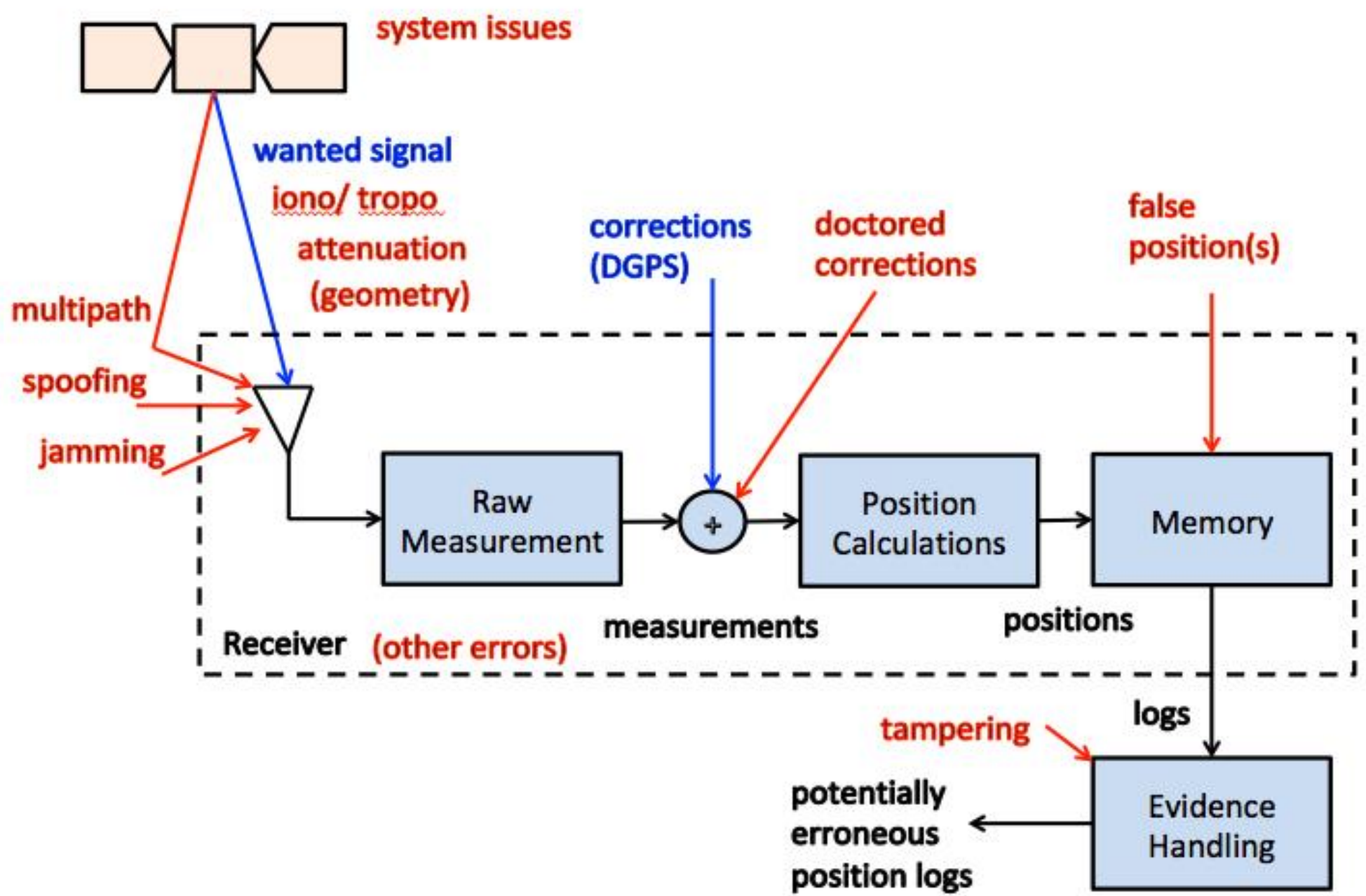


"how accurate was GPS at that time and place?" breaks down into :

1 Was the GPS system operating correctly at that time?

2 Was the ionosphere (and troposphere) behaving itself at that time? If not, was the receiver affected?

3 Was the receiver in a multipath environment? If so, was the receiver affected?

4 Were any of the satellite signals attenuated (e.g. by trees)? If so, was the position calculation affected?

5 Was the receiver jammed or spoofed?

6 Did the position calculation use satellites that had good geometry?

7 Was the position calculation done correctly?

8 Were the data recorded/ communicated/ logged correctly?

9 Was any extra data calculated and/or recorded that gives an indication of accuracy?

10 Were other inputs (other sensors, GPS corrections) used in the position calculation and was this process done correctly?

11 Was the data extracted and presented as evidence without being modified?

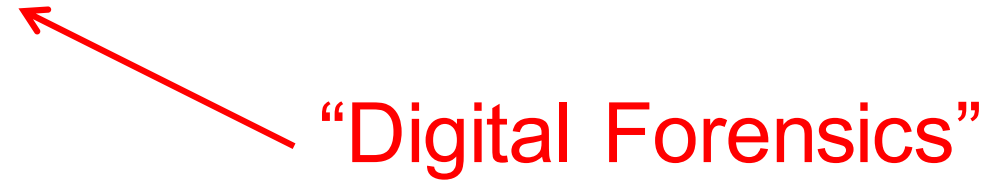




\section{Daubert standard}

[US] Used by trial judge to assess validity of expert witness's evidence

Factors

Has technique in question been tested

Has it been subjected to peer review/publication?

Known potential error rate?

Standards controlling of operation?

Acceptance within relevant scientific community? 


\section{Digital Forensics}

"Criterion 1: Meaning. Has the meaning and, therefore, the interpretation of the electronic evidence been unaffected by the digital forensic process?

Criterion 2: Errors. Have all errors been reasonably identified and satisfactorily explained so as to remove any doubt over the reliability of the evidence?

Criterion 3: Transparency. Is the digital forensic process capable of being independently examined and verified in its entirety?

Criterion 4: Experience. Has the digital forensic analysis been undertaken by an individual with sufficient and relevant experience?" Indrajit Ray and Sujeet Shenoi (eds), "Advances in Digital Forensics IV", International Federation for Information Processing, Slide 16 Springer, New York, 2008, p30 


\section{Example: Securing Evidence}

"Systems which are powered on (running) need to be handled with care, as there is the potential to make unwanted changes to the evidence if these are not dealt with correctly. Such systems should only be accessed by appropriately trained officers...

1. Secure and take control of the area containing the equipment. Do not allow others to interact with the equipment;

2. Photograph the device in situ, or note where it was found, and record the status of the device and any on-screen information;

3. If the device is switched on, power it off. It is important to isolate the device from receiving signals from a network to avoid changes being made to the data it contains. For example, it is possible to wipe certain devices remotely and powering the device off will prevent this.

4. Seize cables, chargers, packaging, manuals, phone bills etc. as these may assist the enquiry and minimise the delays in any examination;

5. Packaging materials and associated paperwork may be a good source of PIN/PUK details;

6. Be aware that some mobile phone handsets may have automatic housekeeping functions, which clear data after a number of days. For example, some Symbian phones start clearing call/event logs after 30 days, or any other user defined period. Submit items for examination as soon as possible." 


\section{Lessons from Remote Sensing}

\section{EVIDENCE FROM} EARTH OBSERVATION SATELLITES

\section{PMERGING LEGAL ISSUES}

PAY FURDY AND DENISI LIUNO 


\section{Part One}

The Scientific, Technological and Policy Context

1. Technical Introduction to Satellite EO .11 Shaida Johnston

2. Science, Policy and Evidence in EO Ray Harris

\section{Part Two}

The Use of EO Data at National Level

3. The Use of Satellite Imagery in Environmental Crimes Prosecutions in The United States: A Developing Area.... .65 Kris Dighe, Todd Mikolop, Raymond W. Mushal and David O’Connell

4. The Use of EO Data As Evidence in the Courts of Singapore........93 Gérardine Goh Escolar

5. Ten Years of Using Earth Observation Data in Support of Queensland's Vegetation Management Framework Bruce Goulevitch

6. EO in the European Union: Legal Considerations .147 Sa'id Mosteshar

7. Satellite Data As Evidence in the Courts of Taiwan .177 Dennis Tsai

\section{Part Three}

The Use of EO Data at International Level

8. Satellite Evidence in International Institutions Maureen Williams

9. The Use of EO Technologies in Court by the Office of the Prosecutor of the International Criminal Court 217 43

\section{Lessons from Remote} Sensing

Part Four

Privacy and Copyright Impacts

10. Outer Space Law Principles and Privacy.

Frans $G$. von der Dunk

11. Privacy and EO: An Overview of Legal Issues George Cho

12. The Impact of Copyright Protection and Public Sector Information Regulations on the Availability of Remote

Sensing Data

Catherine Doldirina

\section{Part Five}

EO Data in the Courtroom: Judicial Perspectives

13. The Use of Remote Sensing Evidence at Trial in the United States-One State Court Judge's Observations Merideth Wright

14. Satellite Images As Evidence for Environmental Crime in Europe: A Judge's Perspective. Carole M. Billiet

Part Six

Trust in and Transparency of EO Data

15. Authentication of Images .359 Alan Shipman

16. Introducing Digital Signatures and Time-Stamps in the EO Data Processing Chain .379 Willibald Croi, Fréderic-Michael Foeteler and Harold Linke 


\section{Scientific, technology and policy context}

EO: UN principles guide behaviour and uses of EO data $\rightarrow$ GNSS has ICG mission and vision statements?

"states have the right to launch and operate EO satellites designed to capture environmental data and those states that are sensed have the right to access the data thereby collected"

EO nation state $v$ nation state; GNSS sensed (i.e. positioned) party an individual, right to sensed data a more local (national, regional) jurisdiction.sidiezo 


\section{Use of GNSS data}

\section{EO data must be authenticated:}

"Evidence is authenticated when testimony establishes that it is real and that it is, or depicts, that which it is purported to be. Federal Rule of Evidence 901 deals with authentication generally, and it requires that a "condition precedent to admissibility" is evidence "that the matter in question is what its proponent claims." 31 Evidence that must be authenticated before it may be admitted includes writings, tangible objects such as guns, and photographs. ${ }^{32}$

${ }^{31}$ Federal Rule of Evidence 902 provides that certain classes of documents are "self-authenticating" and require no further evidence of their authenticity for admission, although there may be other limitations such as relevance. Certain public documents under seal, certified public records, and newspapers fall within the class of self-authenticating documents.

32 The same approach is taken for sound recordings. If a witness testifies that a relevant recording is an accurate reproduction of what he or she heard, it generally will be admitted." 
"A similar approach to authenticate images taken by automated or remotely operated photographic processes has become more accepted. It commonly is called the "silent witness" theory, because there is no live witness to the events captured. Verification of such photographic evidence depends not on a person able to testify that they saw what was captured on film, but on the reliability of the process involved in creating the film. ... courts often will be more comfortable with satellite information that has been verified in some manner. Observations by witnesses, photographs, or other evidence, known as 'ground-truthing,' may be an additional method to not only authenticate the remote satellite imagery, but also provide the court with more familiar forms of evidence that may tend to corroborate the satellite information and make admission more likely" 


\section{Use of GNSS Data}

GNSS "self-authentication" - integrity

GNSS expert witnesses must educate court :"it is the judge's responsibility to see to it that the expert's testimony rests on a reliable foundation and is relevant"

Admissibility of GNSS evidence: future work (different jurisdictions treat it differently, e.g. a "document" or not [16].) 


\section{Simple "Authentication" Example}

The Queen v lan Robert Turnbull, 2014/00223920, NSW Supreme Court.

Question: is the GPS accurate? 


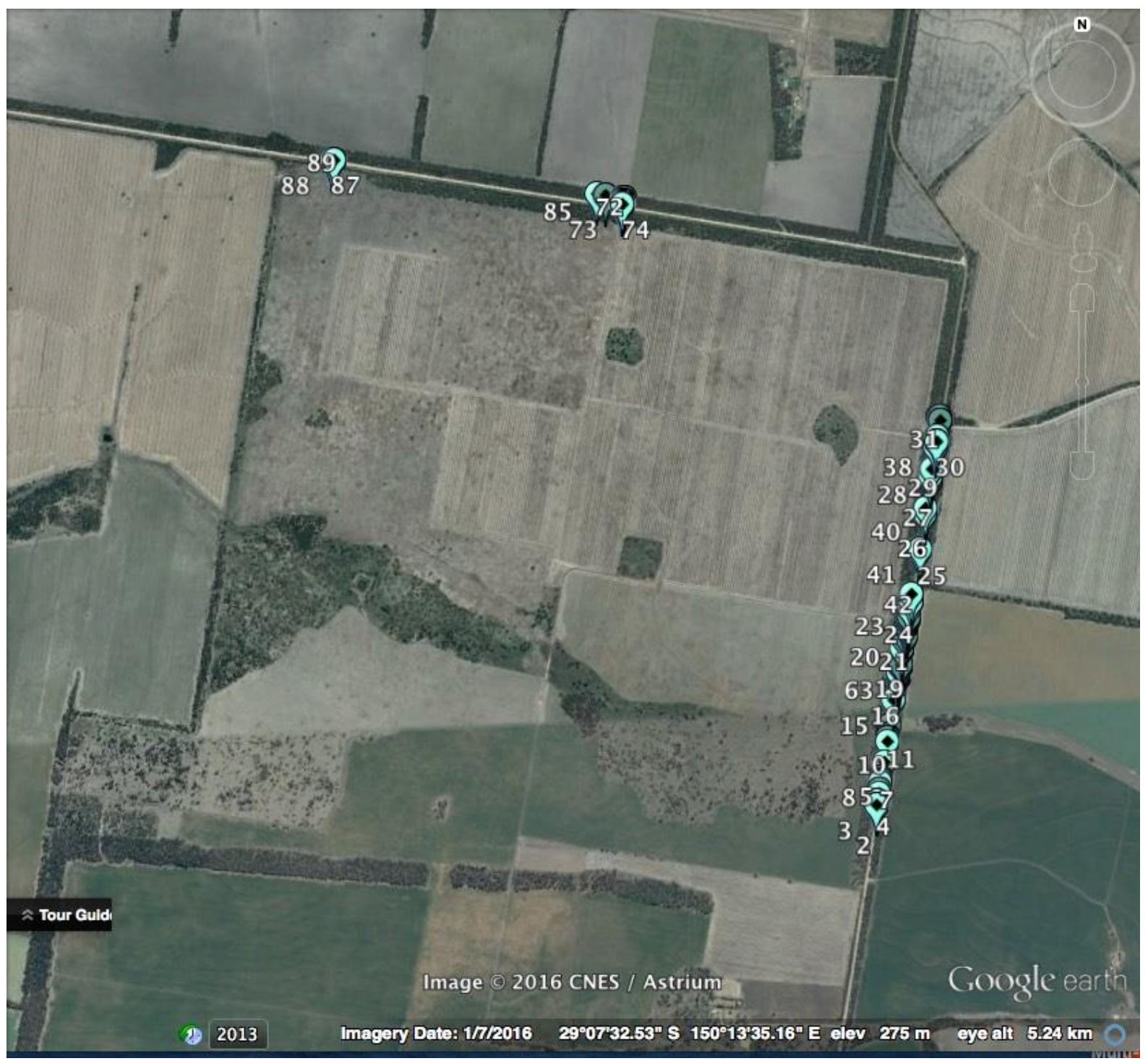

Slide 25 


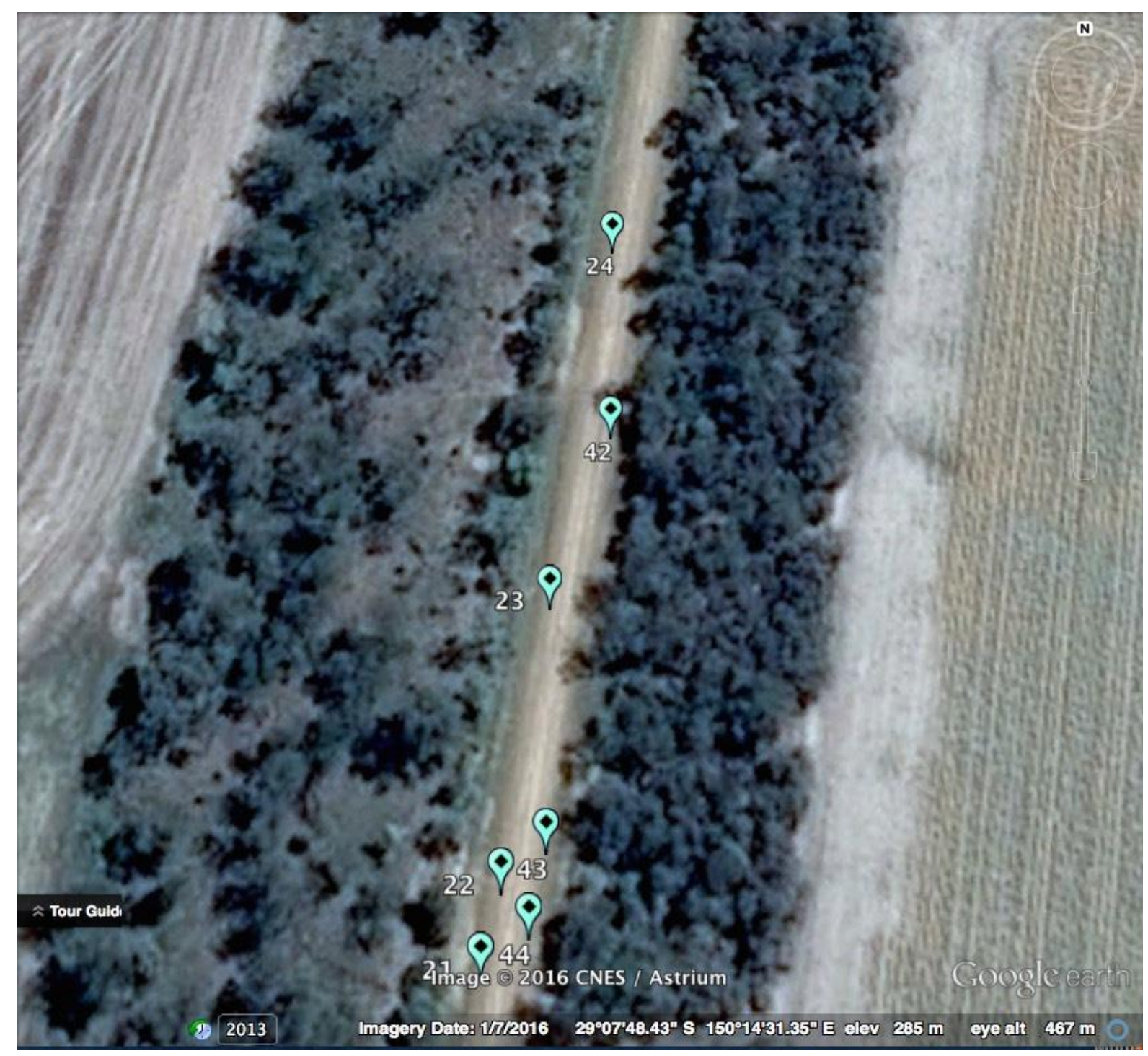

Slide 26 


\section{Privacy and copyright impacts}

Privacy and copyright concerns quite different for EO and GNSS.

Privacy more of an issue for GNSS: personal, ubiquitous (recorded, often without user knowledge), and often made available to purchasers (without the knowledge, and with only dubious consent, of the user).

Copyright less of an issue: EO data product expensive, protected by copyright/ GNSS often not considered a "document". 


\section{GNSS data in the courtroom}

(Authentication again) 


\section{Trust in and transparency of GNSS}

\section{data}

EO evidence can be obscured (e.g. by clouds), have trouble being recorded by the camera (e.g. by single event upsets due to radiation in space) or timing of the image.

Demonstrating evidential weight: creation, transmission and storage. Creation quite different for GNSS

EO imagery governed by, not true for GNSS

Trust in the EO data: digital signatures and time stamps (GNSS logged data?) 


\section{Lessons (for EO community)}

1. Corroborate if possible

2. Build in controls to give greater confidence

3. Ask data suppliers to authenticate the first stage

4. Legislate that the defence must prove incorrect functioning of the system

5. Use systems such as digital signatures to authenticate

6. Follow standards in recording data

7. Write a standard specifically for EO evidence 


\section{Lessons}

GNSS community to write a similar textbook?

Contact me:

a.dempster@unsw.edu.au

\section{EVIDENCE FROM} GLOBAL NAVIGATION SATELLITES

Someone other than Andrew Dempster 


\section{What else can be done?}

Receiver can detect and report on a number of the identified problems:

Attenuated signals

Poor geometry (satellite set enough)

Jamming/ spoofing

Integrity: lono/ Multipath/ Attenuated signal 


\section{Integrity}

e.g. Receiver Autonomous Integrity Monitoring (RAIM) seeks to isolate a poor pseudorange

Originally for a "bad" satellite (led to SBAS), but now any isolated bad measurement 


\section{The RAIM Problem}

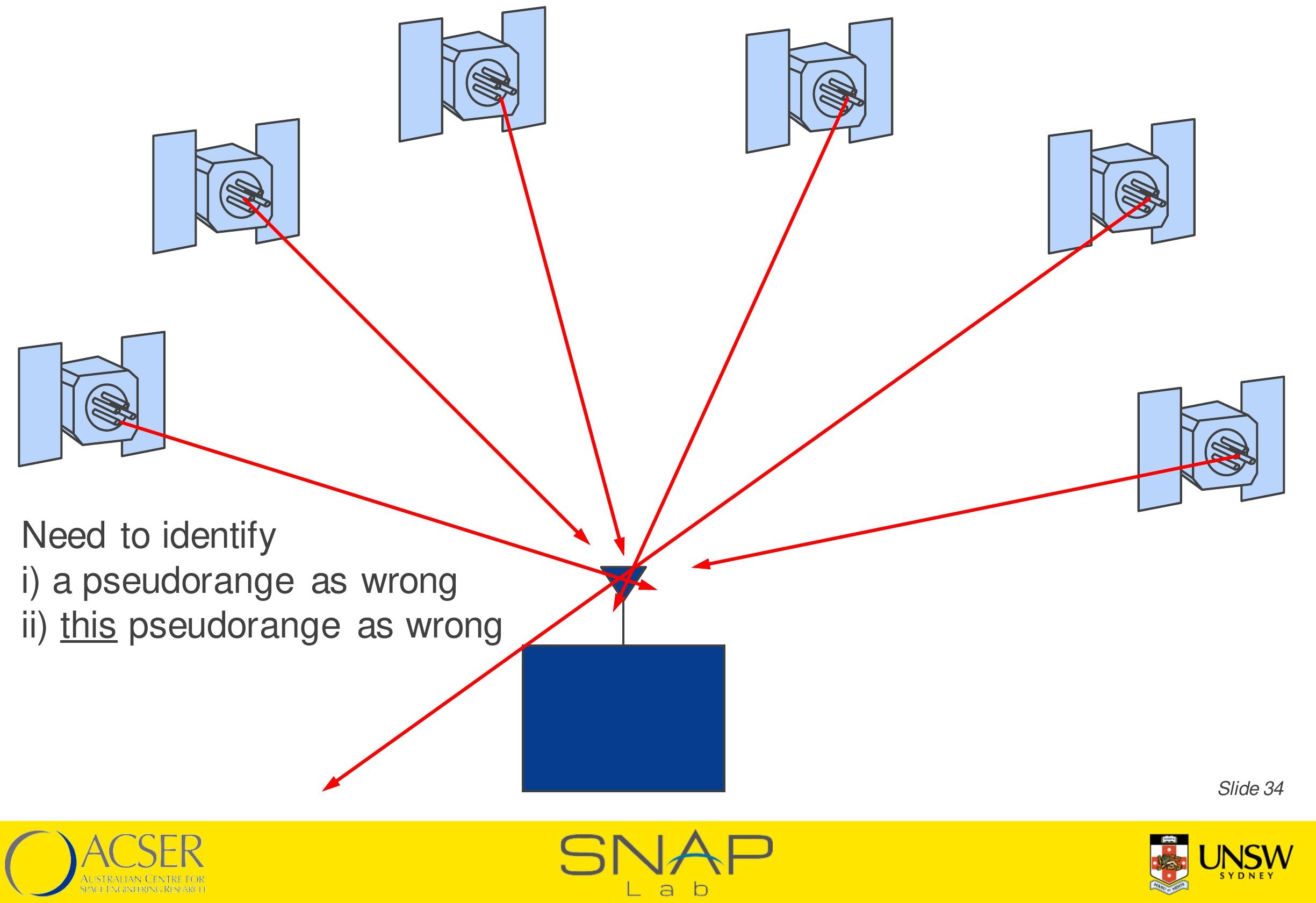




\section{How many Satellites?}

We need redundancy (i.e. > 4 sats), but how many?

If we used 5 , one of which was malfunctioning, we could create 5 sets of 4 sats, 4 of which would contain the malfunctioning sat and produce results inconsistent from each other and the correct set - we couldn't tell which set was right! ie we could tell we had a malfunctioning sat but not which one

Use 6 sats, so there are $C(6,4)=15$ sets of 4 , of which $\mathrm{C}(5,4)=5$ would be consistent 


\section{RAIM: System Requirements}

RAIM is basically a self-consistency check on measurements

Implies redundancy (and good geometry when individual satellites are removed from the set for checking)

Need:

- as many satellites in view as possible

- satellites in good geometry 


\section{RAIM Approaches}

Snapshot:

- only current redundant measurements are used in selfconsistency check

Averaging/filtering schemes

- uses past and present measurements, as well as inferred vehicle motion

Snapshot uses less information but has the advantage of relying only on current information it hasnt used potentially poor information to estimate the current state 


\section{Integrity Terms}

Alert Limit: The alert limit for a given parameter measurement is the error tolerance not to be exceeded without issuing an alert.

Time to Alert: The maximum allowable time elapsed from the onset of the navigation system being out of tolerance until the equipment enunciates the alert.

Integrity Risk: Probability that, at any moment, the position error exceeds the Alert Limit.

Protection Level: Statistical bound error computed so as to guarantee that the probability of the absolute position error exceeding said number is smaller than or equal to the target integrity risk.

(Wikipedia) Slide 38 


\section{Stanford-ESA Integrity Diagram}

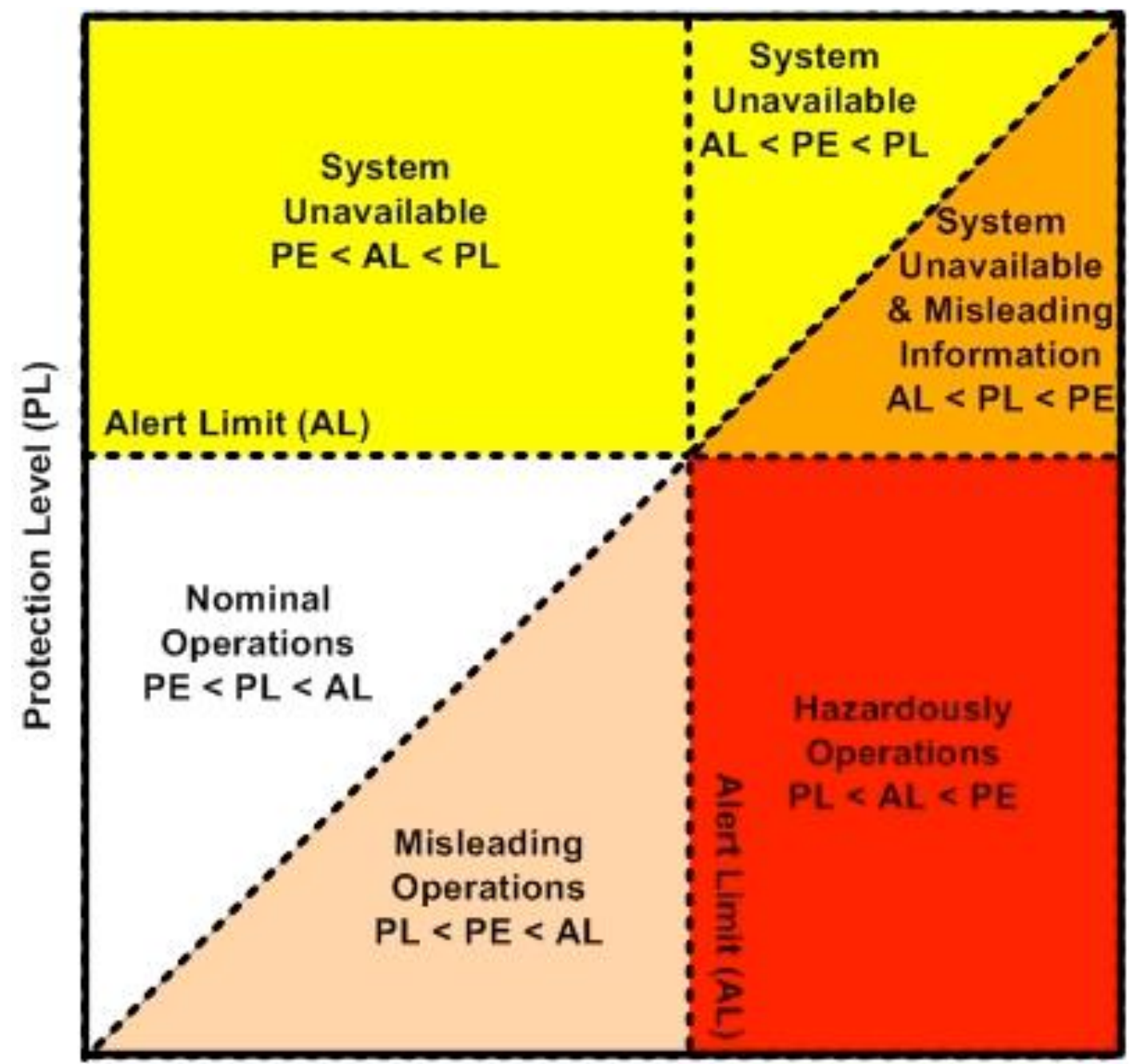

Position Error (PE)

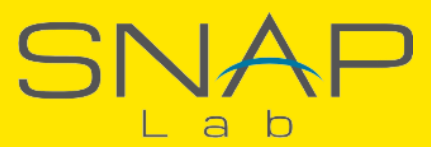




\section{Common Logging Formats}

Can they log useful information?

Do they? 


\section{NMEA Messages: Common}

GGA: time, lat, long, fix quality (GPS, DGPS, PPS, RTK, RTK float, dead-reckoned, manual, or simulated), no. satellites, HDOP, altitude, height of Geoid

GSA: 3D fix, satellites, PDOP, HDOP, VDOP.

GSV: satellites, elevation, azimuth, signal to noise (SNR) 


\section{NMEA Messages: More Useful}

GRS (Range residuals): time, residuals for each satellite.

GST (Pseudorange noise statistics): time, RMS value of residuals, error ellipse semi-major axis, semi-minor axis, orientation, lat 1 sigma, long 1 sigma, height 1 sigma 


\section{Any Other Formats Provide Quality Info?}

GPX: none

Android phones: provide raw range measurements

Aircraft "black box" has no requirement for quality even position!

ADS-B has accuracy and integrity (protection limits)

AIS has position and whether RAIM guarantees

$10 \mathrm{~m}$ accuracy 
Those 11 Questions

\begin{tabular}{|l|l|l|l|l|}
\hline Question & $\begin{array}{l}\text { Source of } \\
\text { Answer }\end{array}$ & $\begin{array}{l}\text { Authenticated } \\
\text { by Other } \\
\text { "Witness"? }\end{array}$ & $\begin{array}{l}\text { Receiver- } \\
\text { Based } \\
\text { Detection? }\end{array}$ & $\begin{array}{l}\text { Integrity } \\
\text { helps? }\end{array}$ \\
\hline GNSS System OK? & GNSS NANU & Y & Y (slow) & Y \\
\hline lono/ Tropo OK? & Networks & Y & Y? & Y \\
\hline Multipath? & Rx & Y & Y & Y \\
\hline Attenuation? & Rx & Y & Y & Y \\
\hline Jam/ spoof? & Rx + & Y & Y & $?$ \\
\hline Geometry OK? & $\begin{array}{l}\text { Rx, post- } \\
\text { processing }\end{array}$ & $\mathrm{Y}$ & $\mathrm{Y}$ & $\mathrm{Y}$ \\
\hline Calcs OK? & Hard & $\mathrm{Y}$ & $\mathrm{Y} ?$ & $\mathrm{~N}$ \\
\hline Recorded OK? & Hard & $\mathrm{Y}$ & $\mathrm{Y} ?$ & $\mathrm{~N}$ \\
\hline Accuracy indicated? & Rx & $?$ & $\mathrm{Y}$ & $\mathrm{Y}$ \\
\hline Other inputs used? & Hard & $\mathrm{Y}$ & $\mathrm{Y} ?$ & $\mathrm{Y} ?$ \\
\hline Extracted OK? & $\begin{array}{l}\text { Digital } \\
\text { forensics }\end{array}$ & $\mathrm{Y}$ & $\mathrm{N}$ & $\mathrm{N}$ \\
\hline J & & & V d \\
\hline
\end{tabular}




\section{Future Work}

Expert witness check list

Recommendations for data logging standard, including a specific integrity indication

Rank evidence types by quality (even if just verbal: poor, moderate, good)

From Solange's work:

- Guidance to the court/ criteria for selection of an expert

- Different types of GNSS data source (covert tracker, data logger, phone, police GNSS)

- Admissibility 


\section{The End}

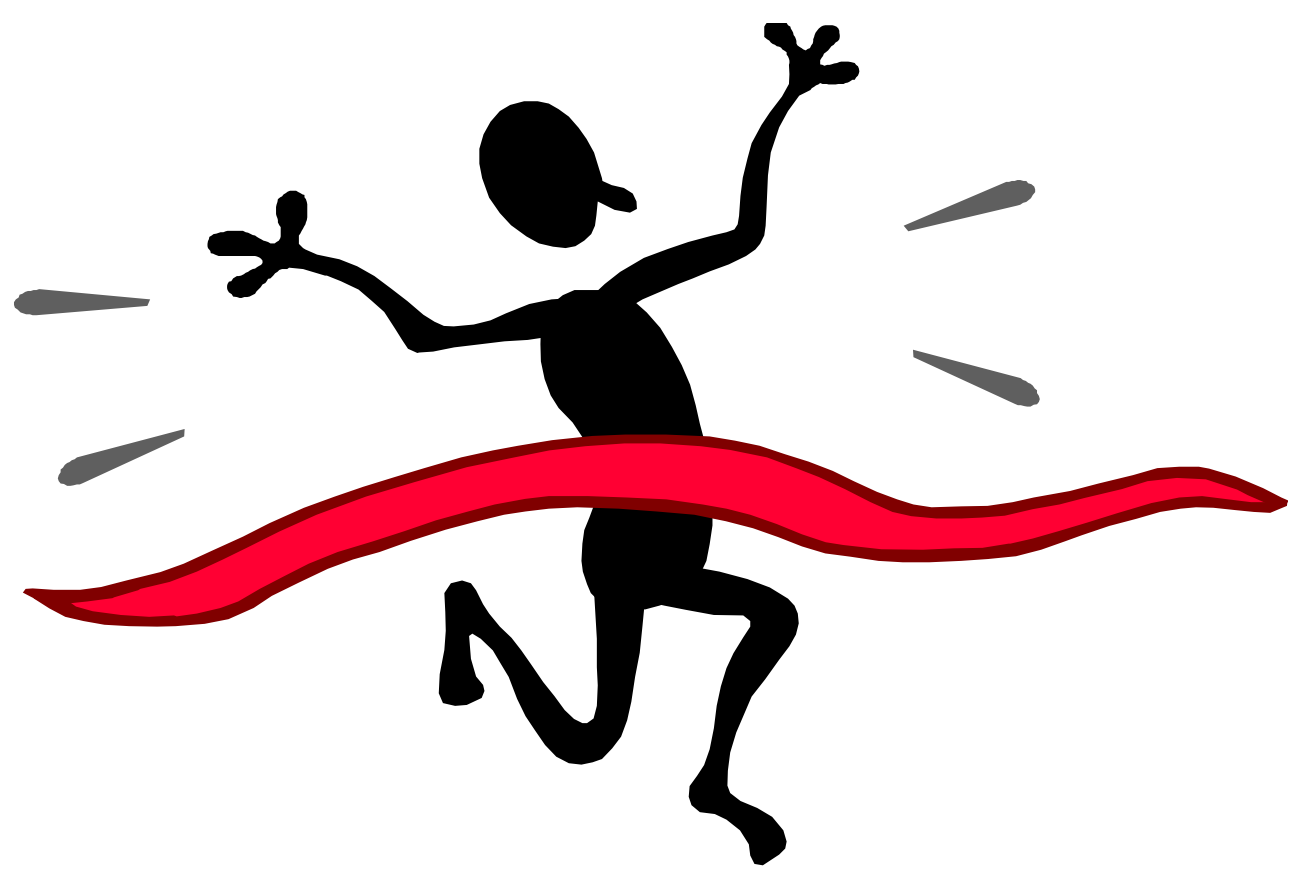

Slide 46

$$
\text { SNAP }
$$




\section{Extra Slides}

Slide 47 


\section{Snapshot Algorithms}

\section{Examine one instant in time}

Methods:

- Range comparison method

- Least-squares residuals method

- Parity Method

Linearise the algebraic problem about a particular position 


\section{Linearised System}

We can define the system as before except we isolate out the pseudorange error:

$$
\Delta \rho=\mathbf{H} \Delta \mathbf{x}+\varepsilon
$$

where $\varepsilon(n \times 1)$ is measurement error vector with usual errors plus malfunctions

However, if solved as before:

$$
\Delta \mathbf{x}=\mathbf{H}^{-1} \Delta \rho
$$

position would incorporate malfunction error 


\section{Range Comparison Method}

Take 4 satellites and perform a linearised solution

Evaluate errors for the extra 2 sats and if either is out of range, signal malfunction

The "out-of-range" threshold is set so that the $1 / 15000$ false alarm rate is achieved 


\section{Least-Squares Residuals method}

$$
\Delta \mathbf{x}=\left(\mathbf{H}^{T} \mathbf{H}\right)^{-1} \mathbf{H}^{T} \Delta \rho
$$

From the least-squares solution, predict the observables:

$$
\Delta \rho_{\text {predicted }}=\mathbf{H} \Delta \mathbf{x}
$$

and difference to get the residuals:

$w=\Delta \rho-\Delta \rho_{\text {predicted }}=\left[\mathbf{I}-\mathbf{H}\left(\mathbf{H}^{T} \mathbf{H}\right)^{-1} \mathbf{H}^{T}\right] \Delta \rho$

from which we get our SSE observable:

$$
S S E=w^{T} w
$$




\section{Least-Squares Residuals method}

\section{For SSE:}

- positive threshold only is needed

- for pseudoranges with the same error statistics, the threshold for a constant alarm-rate algorithm depends only on no. pseudoranges

using a test statistic of $\sqrt{S S E /(n-4)}$ gives linear relationship between statistic and pseudorange bias error (useful as 2D position error is proportional to pseudorange bias error) 


\section{Parity Method}

Set up an equation that uses a matrix $\mathbf{P}$ which has mutually orthogonal rows of unity magnitude, orthogonal to the columns of $\mathbf{H}$ :

$$
\left[\begin{array}{c}
\Delta \mathbf{x} \\
\mathbf{p}
\end{array}\right]=\left[\begin{array}{c}
\left(\mathbf{H}^{T} \mathbf{H}\right)^{-1} \mathbf{H}^{T} \\
\mathbf{P}
\end{array}\right] \Delta \rho
$$

This produces parity vector $\boldsymbol{p}$ (two elements if 6 sats in set)

Special properties of $\mathbf{p}$ ensure

$$
\mathbf{p}^{T} \mathbf{p}=w^{T} w=S S E
$$




\section{Maximum Separation of Solutions}

\section{method}

Assume no more than one satellite failure

Evaluate all solutions, leaving one sat out

If pair of solutions is more than $200 \mathrm{~m}$ apart, failure

Protects against $300 \mathrm{~m}$ errors, assuming $100 \%$ of errors < 100m (actually 95\%)
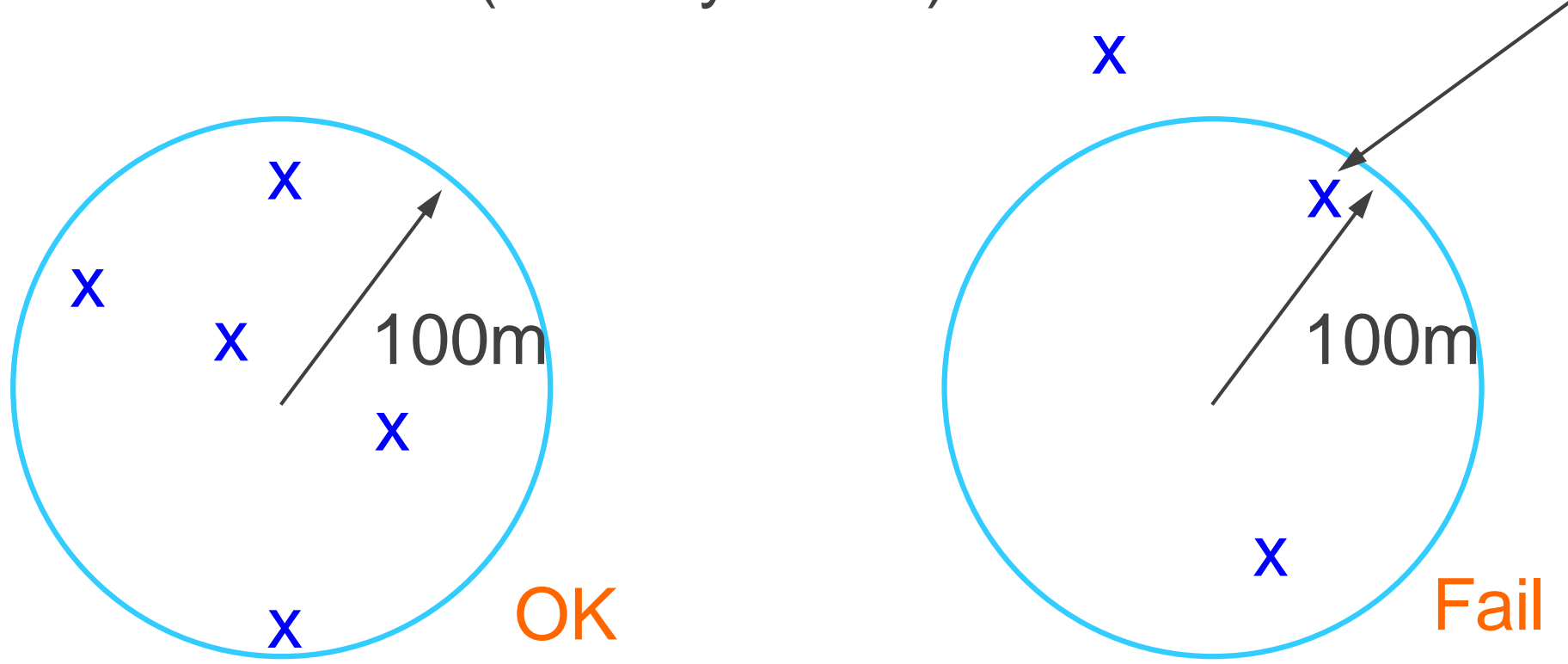


\section{Eliminating Poor Sets}

Sets with poor geometry must be marked inadmissible

May still give a good position but there is insufficient redundancy to ensure integrity

Must be careful eliminating poor geometry, as any periods of inadmissibility reduce system availability 


\section{Inadmissibility: HDOP $_{\text {max }}$}

DOP is a reasonable measure of geometry (as it is for positioning) because if DOP is too high, we know that it is difficult to identify an error in pseudorange

Check each set with one satellite missing and if HDOP $_{\text {max }}$ is above a threshold, mark the complete set as inadmissible (i.e. can't be relied upon for integrity)

Only a coarse measure of geometry quality 


\section{Inadmissibility : $\delta \mathrm{H}_{\max }$}

Evaluate $\mathrm{HDOP}_{\mathrm{i}}$ for the $\mathrm{n}$ subsets

Evaluate HDOP for the complete set

$\delta \mathrm{H}_{\max }=\max _{\mathrm{i}}\left[\mathrm{HDOP}_{\mathrm{i}}{ }^{2}-\mathrm{HDOP}^{2}\right]^{1 / 2}$

Threshold $\delta \mathrm{H}_{\max }$

Gives more reliable result than $\mathrm{HDOP}_{\text {max }}$ 


\section{Failure Isolation}

So far, all techniques have been for malfunction detection

OK where a backup nav system exists, but no good for sole-means

Failure Detection and Isolation (FDI) 


\section{FDI: Parity method}

Remember the parity vector $\mathbf{p}$ which indicated malfunction:

$$
\left[\begin{array}{c}
\Delta \mathbf{x} \\
\mathbf{p}
\end{array}\right]=\left[\begin{array}{c}
\left(\mathbf{H}^{T} \mathbf{H}\right)^{-1} \mathbf{H}^{T} \\
\mathbf{P}
\end{array}\right] \Delta \rho
$$

The orthogonality property of $\mathbf{P}$ ensures:

$$
\mathbf{p}=\mathbf{P} \Delta \rho=\mathbf{P}(\mathbf{H} \Delta x+\varepsilon)=\mathbf{P} \varepsilon
$$

i.e. $\mathbf{P}$ projects the error onto $\mathbf{p}$. If there is a bias $b$ in pseudorange $i$,

i.e. a point on a line with slope $p_{2 i} / p_{1 i}$

$$
\mathbf{p}=\left[\begin{array}{l}
p_{1 i} \\
p_{2 i}
\end{array}\right] b
$$




\section{FDI: Parity method}

Each satellite has a unique line associated with it

Rule: select line closest to $\mathbf{p}$

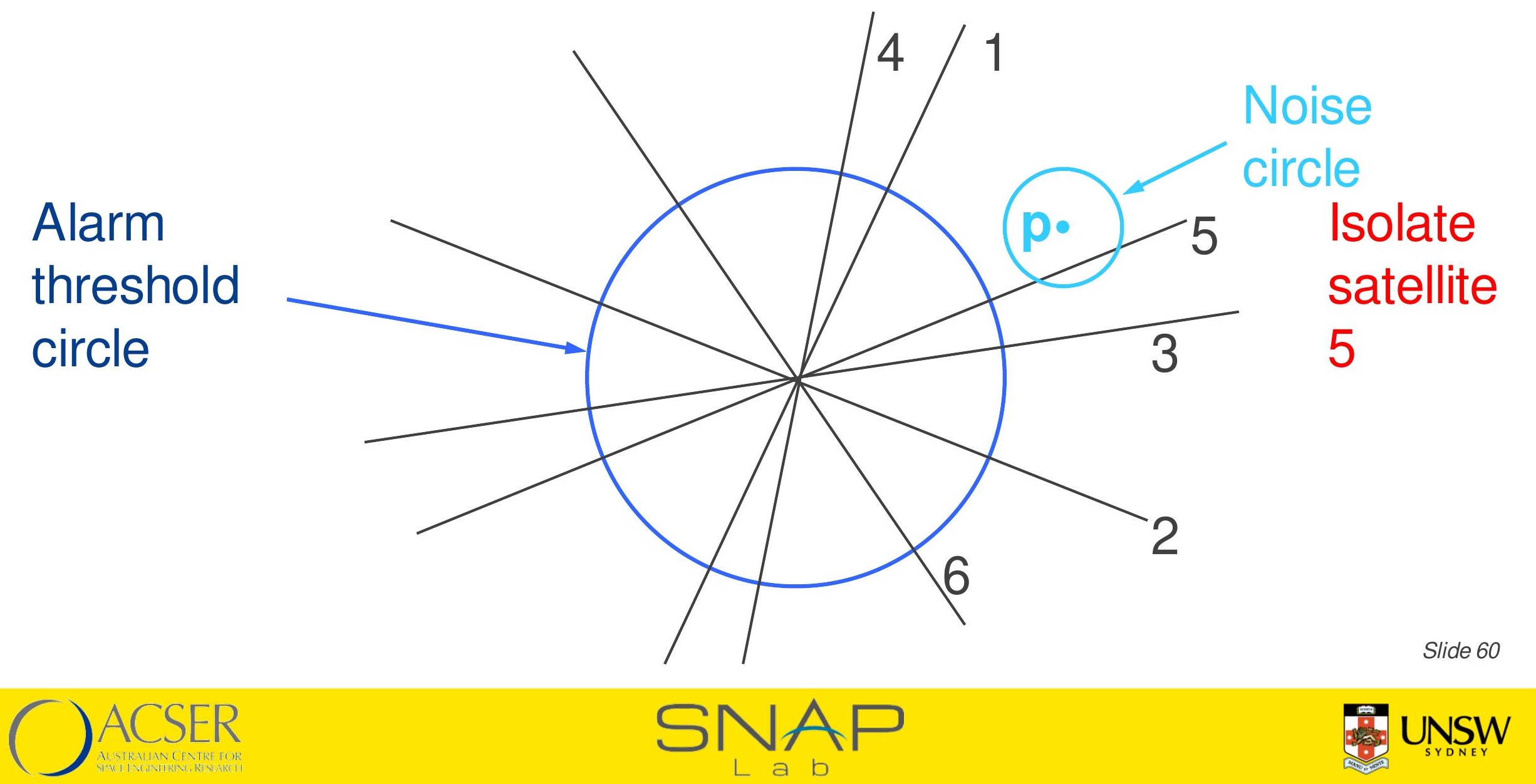




\section{References}

B W Parkinson and J J Spilker Jr., "Global Positioning System: Theory and Applications", vols I \& II, American Inst Aeronautics \& Astronautics, 1996 